\title{
Size-Sorting and Pattern Formation of Nanoparticle-Loaded Micellar Superstructures in Biconcave Thin Films
}

\author{
Jan Bart ten Hove, ${ }^{\dagger, \ddagger 0}$ Junyou Wang, ${ }^{\dagger}$ Matthias N. van Oosterom, ${ }^{\ddagger}$ Fijs W. B. van Leeuwen, ${ }^{\dagger, \ddagger}$ \\ and Aldrik H. Velders $*, \dagger, \$, \delta(0)$
}

${ }^{\dagger}$ Laboratory of BioNanoTechnology, Wageningen University \& Research, Axis, Bornse Weilanden 9, 6708 WG Wageningen, The Netherlands

${ }^{\ddagger}$ Interventional Molecular Imaging Laboratory, Department of Radiology, Leiden University Medical Centre, 2300 RC Leiden, The Netherlands

${ }^{\S}$ Instituto Regional de Investigacion Cientifica Aplicada, Universidad de Castilla-La Mancha, 13071 Ciudad Real, Spain

\author{
Supporting Information
}

ABSTRACT: Biconcave thin water layers represent a template to induce organization of supramolecular structures into ordered monolayers. Here we show how micelles form extensive micrometer-sized pseudo-2D superstructures that reveal size-sorting and geometric pattern formation, as shown by cryo-transmission electron microscopy (cryoTEM). Electron-rich gold particles inside the micelles facilitate direct visualization and determination of size, composition, and ordering of the micellar assemblies over multiple length scales. Some of the patterns observed show intriguing geometric patterns for superstructures, including Fibonacci-like, double-spiral domains that also appear in, for
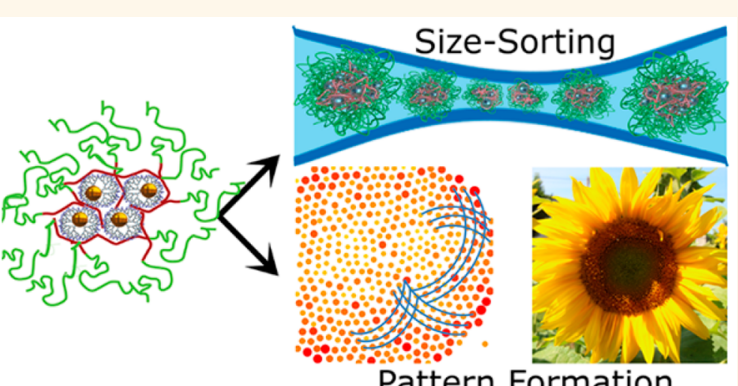

Pattern Formation example, sunflower seed head patterns.

KEYWORDS: dendrimers, self-assembly, micelles, superstructures, size-sorting, biconcave template, thin films

$\mathrm{T}$ he enigmatic symmetry and ordering observed in biological systems has raised wonder and interest since mankind started observing nature, as described first in great detail by D'Arcy Thompson in 1917 in his seminal book On Growth and Form. ${ }^{1}$ Order plays an important role at extreme length scales, also in nonbiological systems, from atomic ordering in crystals to spiral structures in galaxies light years in diameter. ${ }^{2,3}$ Organization is also at the heart of sciences at the nanoscale, such as self-assembly of (macro)molecules and nanoparticles, where specific supramolecular interactions can generate complex yet discrete superstructures. ${ }^{3-9}$ However, it is still a great challenge to control and investigate composition and ordering of hierarchically built-up systems over multiple length scales; micelles provide a versatile tool for such investigation.

Coacervate core micelles are nanoassemblies in which oppositely charged polymeric building blocks form the micelle core and neutral polymer blocks constitute the micelle corona. Many micelle building blocks have been reported, including proteins and dendrimers. ${ }^{10,11}$ Dendrimers are well-defined hyperbranched macromolecules with the potential to encapsulate molecules or nanoparticles, with polyamidoamine (PAMAM) dendrimers being among the most studied. ${ }^{12,13}$ In the past, we showed that carboxyl-terminated, negatively charged, PAMAM dendrimers are versatile and robust micellar building blocks, ${ }^{14}$ and more recently we showed that amineterminated, positively charged PAMAM dendrimers also form well-defined dendrimicelles through coacervation with charged block copolymers. ${ }^{15}$ In PAMAM dendrimers small nanoparticles can be encapsulated, ${ }^{16-18}$ which we managed to incorporate into dendrimicelles. The electron-rich, nanometersized, gold nanoparticles in the core of these micelles permit direct visualization and determination of size and composition of the 50-nm-sized nanoassemblies, by cryoTEM. We here present detailed cryoTEM analysis in which the micelles form extensive, ordered monolayer superstructures in the biconcave thin water layer.

Figure 1 shows this 4-fold organization of our dendrimicelle system, where nanometer-sized particles, templated in and residing inside dendrimers and assembled in micellar core structures, organize into well-defined micrometer-sized super-

Received: August 4, 2017

Accepted: November 1, 2017

Published: November 1, 2017 

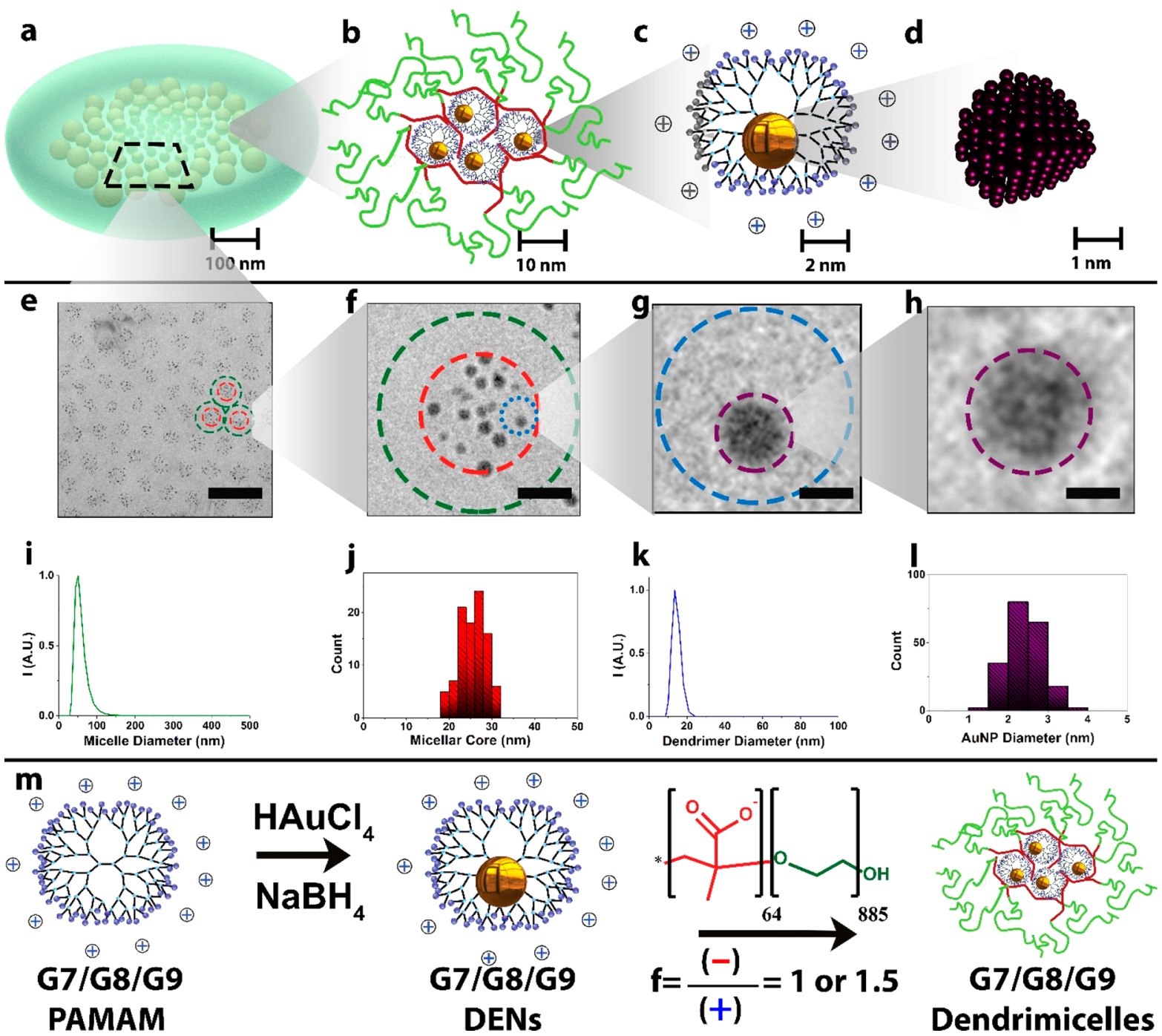

Figure 1. Organization and ordering over multiple length scales. (a) Illustration of a cryoTEM-grid hole consisting of a thin layer of water with patterns of assembled (b) complex coacervate core micelles, consisting of a negative (red) - neutral (green) $\mathrm{pMAA}_{64}-b$-PEO ${ }_{885}$ diblock copolymer and (c) a positively charged (blue) PAMAM core with an encapsulated (d) gold nanoparticle. The dendrimer-encapsulated nanoparticles provide excellent contrast for cryoTEM (scale bars in $\mathrm{e}-\mathrm{h}$ correspond to the ones in $\mathrm{a}-\mathrm{d}$, respectively). DLS reveals (i) micelles with a hydrodynamic diameter of about $50 \mathrm{~nm},(\mathrm{j})$ with a core of about $25 \mathrm{~nm}$ as derived from TEM. In (e) and (f), the green circles indicate the dendrimicelle hydrodynamic diameter as determined by DLS, and the red circles indicate the dendrimicelle core size as obtained from the cryoTEM micrographs. The hydrodynamic diameter of the eighth-generation PAMAM is about $14 \mathrm{~nm}$, as determined by DLS ( $\mathrm{k}$ ), and the gold nanoparticles inside the dendrimer are about $2.4 \mathrm{~nm}(\mathrm{l}) .(\mathrm{m})$ Scheme showing the synthesis of nanoparticle-containing dendrimicelles. Complexation and reduction of gold(III) ions in PAMAM dendrimers yield dendrimer-encapsulated nanoparticles. Addition of pMAA $_{64}-b$ $\mathrm{PEO}_{885}$ results in dendrimicelle formation. The blue circle in $(\mathrm{g})$ indicates the dendrimer size as determined by DLS, and the purple circles in (g) and (h) indicate the AuNP.

structures in biconcave thin water films. As dendrimers can be considered molecular boxes and micelles as nanoboxes, we coined the composite structure a [particle-in-a-box]-in-a-box nanostructure, reflecting back on the two separate levels of hierarchy of encapsulation. The first level concerns the nanoparticle encapsulation inside the dendrimer box, and the second level the encapsulation (of many of these dendrimer boxes) inside the micelle box. Our strategy allows for the straightforward variation of subcomponents, to tune size and polydispersity of the micelles. We here present superstructures from micelles obtained with three different generations of dendrimers and with two different subcomponent ratios to vary polydispersity. The use of the nanoparticles residing at the inside of one of the assembled components reveals composition, ordering, and patterns over multiple length scales.
These superstructures reveal size sorting of the micelles within the superstructures and the formation of geometric patterns, including Fibonacci-like double-spiral domains resembling those that appear in, for example, sunflower seed heads and pine cones. ${ }^{1,2,19,20}$ As the ordering in natural systems is called phyllotaxis, we coin the ordering of nanoparticles at the (sub)micrometer scale nanotaxis.

\section{RESULTS AND DISCUSSION}

Dendrimer-encapsulated gold nanoparticles, Au-DENs, were synthesized following the original work of Amis by reduction of gold(III) ions residing inside PAMAM dendrimers ${ }^{16,21}$ and as reported for generation 7 dendrimers elsewhere. ${ }^{15}$ We focused on generations 7,8 , and 9 in order to have single particles of respectively $\mathrm{Au}_{256}, \mathrm{Au}_{512}$, and $\mathrm{Au}_{1024}$ per dendrimer and 

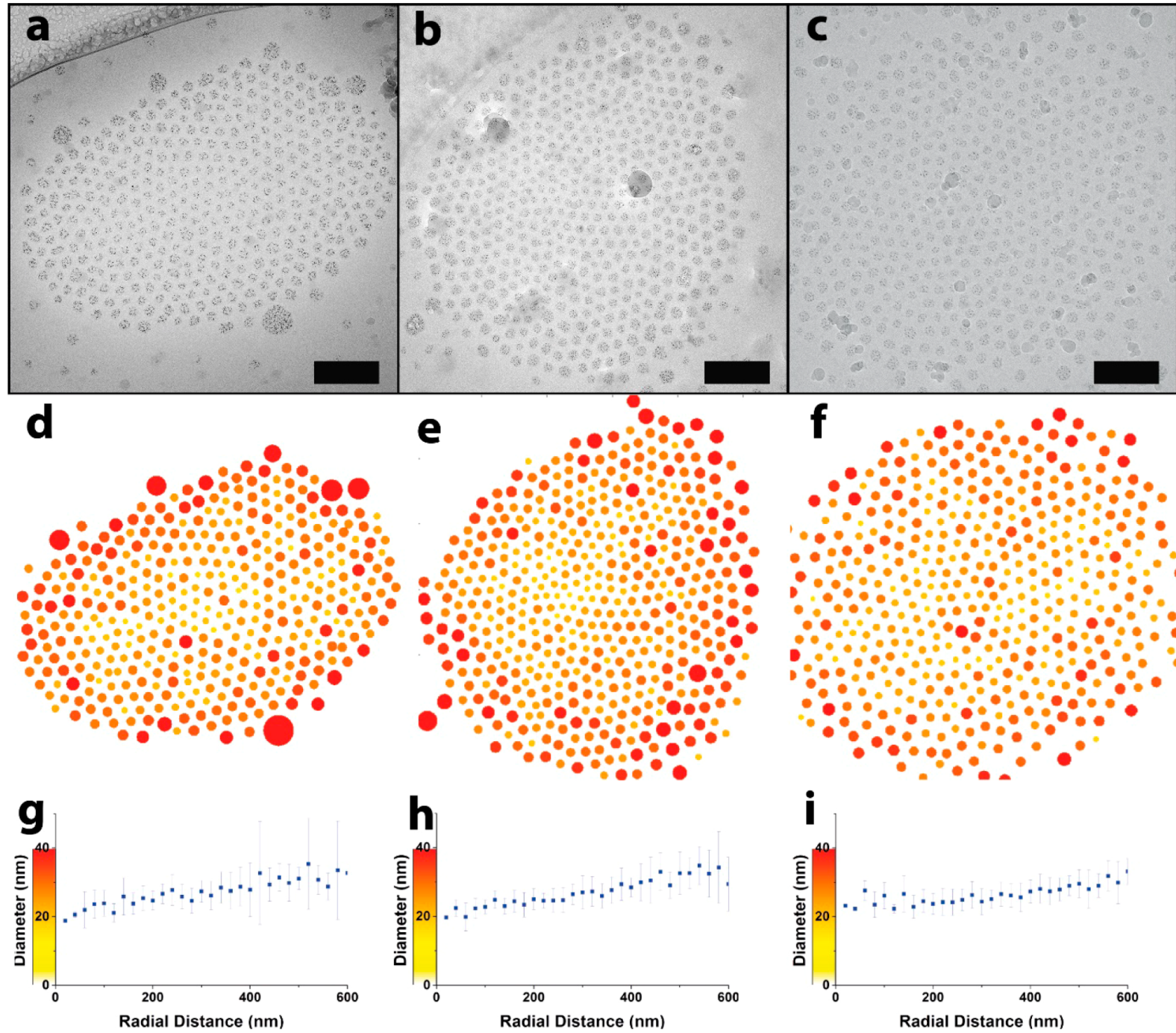

Figure 2. Ordering of seventh (left), eighth (middle), and ninth (right) generation PAMAM dendrimicelles. (a-c) The darker spots in the cryoTEM images are the $2-3 \mathrm{~nm}$ gold nanoparticles residing inside the dendrimers in the micelle core; scale bars correspond to $200 \mathrm{~nm}$. (df) Image segmentation results in heat map plots with micelle cores color-coded according to their size. (g-i) Radial distance plots show micelle diameter versus distance to the center of the pattern.

aggregation numbers in the dendrimicelles between 10 and 40, to allow straightforward discrimination and counting of individual nanoparticles. TEM analysis showed the formed Au-DENs to be about $2-3 \mathrm{~nm}$ in diameter, with the PAMAM ranging in hydrodynamic diameter (dynamic light scattering, DLS) from 9.6 to $17 \mathrm{~nm}$ for generation 7 to 9 (Figure S1). Consecutive mixing under charge stoichiometry conditions (Figure S2) of the Au-DENs with a $\mathrm{pMAA}_{64}-b-\mathrm{PEO}_{885}$ block copolymer, consisting of a 64-subunit polymethacrylic acid block and an 885-subunit poly(ethylene oxide) (PEO) neutral block, resulted in coacervate-core micelle formation as proven by DLS.

The cryoTEM micrographs (Figure 1) of a sample obtained from generation $8 \mathrm{Au}$-DENs clearly corroborate formation of dendrimicelles, with a core size of about $25 \mathrm{~nm}$ as deduced from the gold particles delineating the core; the PEO corona is not observed, but its size can be deduced from the intercore distances to be about $7 \mathrm{~nm}$. The average micelle diameter of about $40 \mathrm{~nm}$, as determined from TEM, is slightly smaller than the about $50 \mathrm{~nm}$ hydrodynamic diameter obtained from DLS, which could indicate the PEO chains to be compressed or even interpenetrating with neighboring micelles upon packing in the superstructure. ${ }^{22}$ Counting the Au-DENs per micelle core allows a straightforward determination of the aggregation number of the components and hence the size of the whole micelle. For the system shown in Figure 1, on average $27 \mathrm{Au}$ DENs reside inside a micelle, corresponding to 27 PAMAM dendrimers and about 400 block copolymers at charge stoichiometric mixing conditions. The total molecular dry weight of such micelles is therefore on the order of about 25 MDa. We note that cryoTEM micrographs are the $2 \mathrm{D}$ representation (image) of the $3 \mathrm{D}$ system (the micelle), and therefore, particles that are overlapping in the micelle shown in Figure If are not fused; they just have the same $x, y$-coordinates but are separated along the $z$-axis. The packing fraction of the DENs in the dendrimicelle core is $\sim 0.9$, indicating that the dendrimers are densely packed. Consequently, the interparticle distances in the dendrimicelle core range between $\sim 3$ and $\sim 15$ $\mathrm{nm}$ (Figure S3). While analyzing the dendrimicelles in the cryoTEM micrograph, we observed ordering and organization on the micrometer scale, suggesting the formation of monolayer superstructures. 


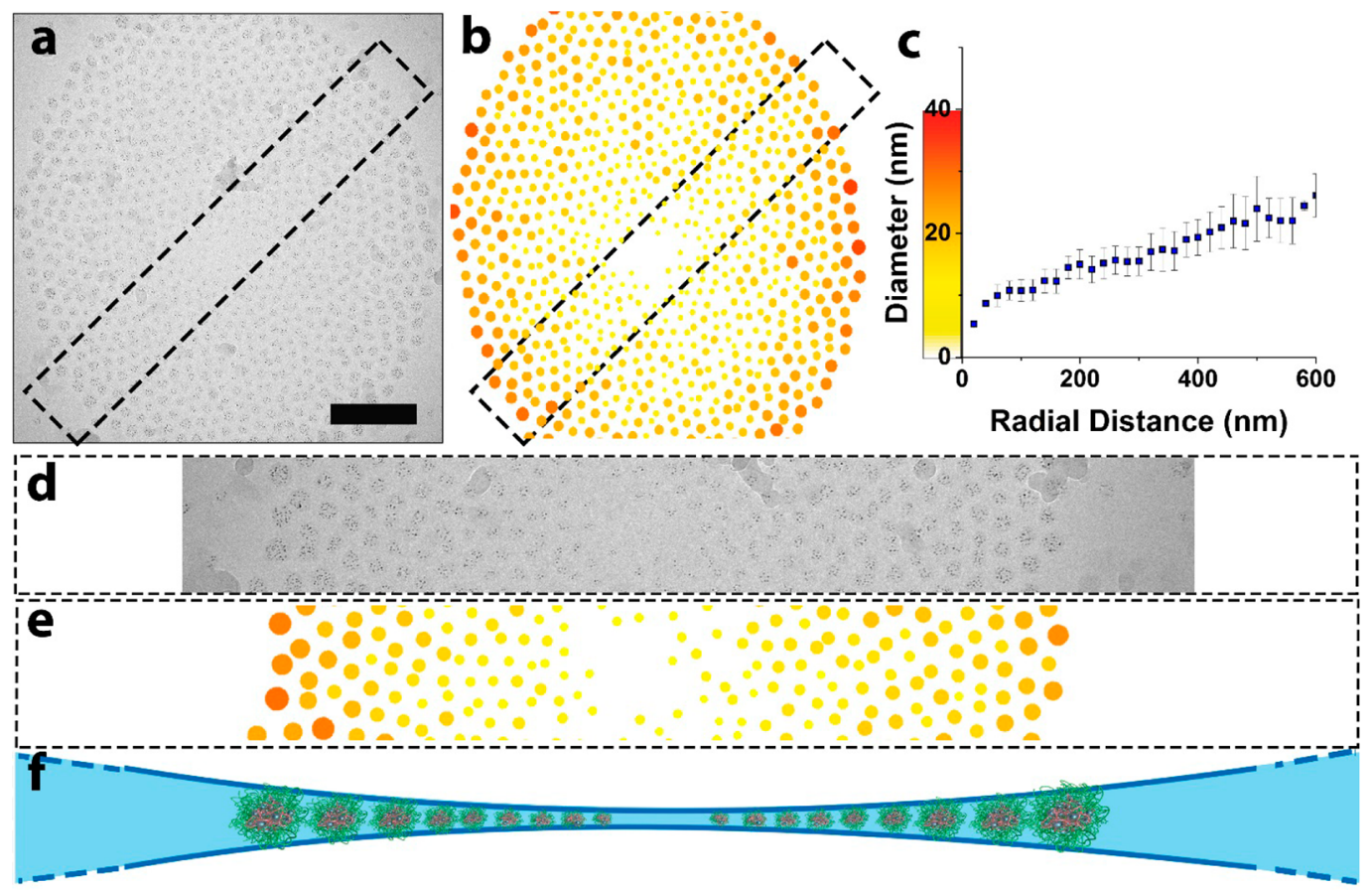

Figure 3. Extreme size sorting of dendrimicelles in biconcave thin films. CryoTEM micrograph (a) of a superstructure obtained from G7based dendrimicelles obtained at noncharge stoichiometric conditions $(f=1.5)$. Color- and size-coded heat map highlighting the size sorting (b). Core diameter of the dendrimicelles versus radial distance from the center of the superstructure. Slice from the cryoTEM micrograph (d) and corresponding heat map (e) visualizing the extreme size sorting. Side-view impression of the distribution of differently sized micelles in the thin biconcave water layer in a TEM-grid hole.

To investigate the effect of the dendrimicelles on the higherorder organization, we prepared a series of dendrimicelles made from different dendrimer generations and studied these with cryoTEM. Representative cryoTEM micrographs of the series of dendrimicelles based on Au-DENs of generation 7, 8, and 9 PAMAM are shown in Figure $2 \mathrm{a}-\mathrm{c}$. The micrometer-sized grid holes contain several hundred micelles, which organize by size and into domains with discrete geometric patterns. Image analysis allowed for particle tracking and segmentation of individual micelles and was subsequently used to graphically display the positions and size of the micelles, with color coding highlighting the small size differences observed (Figure 2d-f). The color coding also facilitates the discrimination of a clear size sorting in the patterns, with the smaller micelles being positioned more toward the center of the circular superstructures and the bigger ones more toward the outside. The vitreous ice layer formed in the holes of the TEM grid foil is biconcave shaped and thinner toward the center. ${ }^{23-25}$ Figures S4-6 show average image intensity plots as derived from the TEM micrographs, which are indicative for the thickness of the vitreous ice layer. These figures confirm that the ice layer is thinner toward the center of the dendrimicelle superstructure and give an estimate of the curvature of the biconcave thin film.

Plotting the determined micellar core diameter against the distance from the center of the superstructure further corroborates the size sorting (Figure $2 \mathrm{~g}-\mathrm{i}$ ) and shows the micelles evidently sort according to the thickness of the water layer. Additionally, clear domains with different geometric patterns are observed for all three different dendrimicelle systems (Figures S7-9) and appeared in samples prepared on different occasions and with different preparation protocols. CryoTEM provides snapshots of the micelle organization process during and after the blotting step, which does not necessarily represent a thermodynamic equilibrium state of the dendrimicelles in the thin film. The formation of the water layer in the blotting process prior to vitrification inflicts dramatic forces on the sample. In fact, the micrometer-size circular areas observed often consist of multiple domains (Figures S7-9 for highlighted domains) and characteristic geometric patterns and five and seven nearest neighbor sites (Figures S10-12).

The size sorting of dendrimicelles in the biconcave thin water film was further investigated with dendrimicelle samples obtained under noncharge stoichiometric conditions, i.e., with a 1.5 times excess of block copolymer. The resulting dendrimicelles were expected to be more polydisperse and show different packing and size sorting in the formed superstructures. Indeed, all three dendrimicelles, obtained from G7, G8, and G9 DENs, show increased size-sorting features (see Figures S13-19). The most prominent example was obtained from a G7 dendrimicelle, shown in Figure 3. The TEM micrograph and the generated color-labeled heat map plot show a dramatic size sorting (Figure $3 \mathrm{a}-\mathrm{c}$ ). A cross section of the superstructure further highlights this sorting of dendrimicelles with sizes that range in the core diameter from 10 to $20 \mathrm{~nm}$. The size sorting is most likely templated by the thickness of the thin water film formed during sample preparation, which is a double, biconcave, air-water interface that becomes thinner toward the center (see Figure $3 \mathrm{f}$ ). We note that when this layer is too thick, we do not detect the formation of the superstructures nor observe size sorting and only witness dendrimicelles that are randomly distributed throughout the TEM grid hole.

Monolayer superstructures typically form as real $2 \mathrm{D}$ structures on flat surfaces, at solvent--air interfaces, or on curved surfaces. $^{26-31}$ The biconcave thin water layer formed in the cryoTEM preparation provides a double air-water curved 

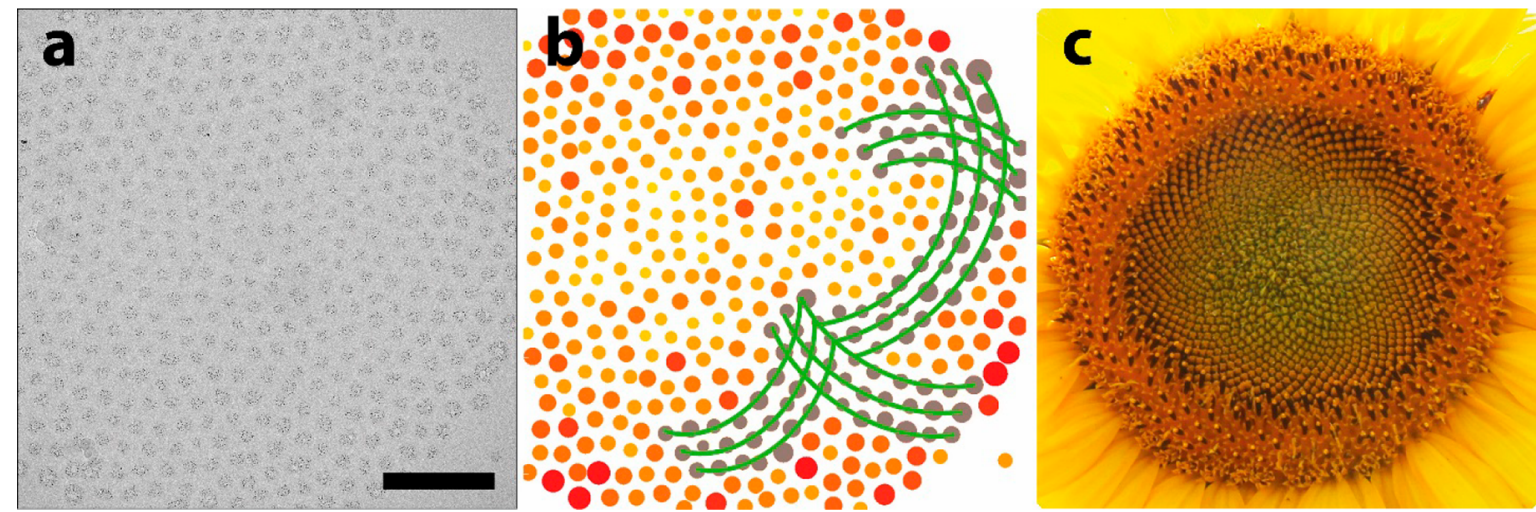

Figure 4. Double-spiral sunflower-like patterns in dendrimicelle superstructures. (a) CryoTEM shows large multiple spiral domains; scale bar corresponds to $200 \mathrm{~nm}$. (b) Corresponding color-coded heat maps highlighting geometric features such as intersecting spirals, a feature commonly observed in nature, e.g., (c) sunflower heads, with Fibonacci-related symmetry.

interface where the micelles are likely arranging symmetrically in the layer, touching both interfaces. Interestingly, the thin water layer in the center of the superstructure shown in Figure 3 does not contain any dendrimicelles, indicating the layer to be thinner than $20 \mathrm{~nm}$ and possibly just a few nanometers in the center. Figure S14 shows the average intensity plot over the superstructure, corroborating the size sorting observed with the ice layer thickness.

The size-sorting and pattern formation have been further investigated in detail for a generation 7 PAMAM-based dendrimicelle with particularly large domains. Figure $4 \mathrm{a}$ shows a cryoTEM image with Au-DENs revealing domains hundreds of nanometers across containing multiple spiral geometries (see Supplementary Figures S20-22 for enlarged figures). Segmentation of the TEM micrograph allowed reconstruction of the image, showing interesting double-spiral domains, of which a few have been highlighted (Figure 4b). Similar Fibonacci-related spiral patterns and symmetry are commonly observed in nature at millimeter, centimeter, or even larger scales, ${ }^{1-3}$ e.g., the archetypical sunflower seed head (Figure 4c). More recently, such geometries have been discovered to form in stress-induced micrometer-sized coreshell particles ${ }^{28}$ and Fibonacci-related ordering is observed also in $2 \mathrm{D}$ quasicrystals. ${ }^{32}$ The spiral patterning features observed for the micelle superstructures in the biconcave thin water layer template is further extending this extraordinary symmetry into the nanoscale.

\section{CONCLUSIONS}

The micelle strategy presented here offers stable structures (Supplementary Figure S23) with a great potential for further generating and understanding hierarchically orchestrated [particle-in-a-box]-in-a-box superstructures, via changing the nanoparticle or dendrimer loading, using different types of dendrimers or micelles, or with different or even mixed components. Finally, the ordering is not expected to be limited to micelle structures, but might be well observed for other (bio)macromolecular and/or supramolecularly assembled systems. The biconcave water layer that progressively becomes thinner in the cryoTEM sample preparation induces physical stress on the sample, which together with the complex interplay of attractive and repulsive forces between the micelles, solvent, and air at the water-air interfaces eventually results in assembly and formation of the superstructures. For 3D systems, the packing of soft nanoparticles has been investigated and modeled in great detail. ${ }^{33}$ Paradoxically, 2D systems, like the superstructures presented here, are of a very different complexity, in which not only interparticle forces play a role, ${ }^{34}$ but also polydispersity and size gradients, air-solvent interfaces, and reciprocal template effects play crucial roles. In addition, the deformability of micelles under stress could also play a role, as particle shape has shown to be of influence for packing as well. ${ }^{27}$ Finally, relatively large domains are observed, yet the presence of multiple domains within one superstructure indicates the pictures obtained are snapshots of out-ofequilibrium situations. It is experimentally a challenge to get control on all parameters involved in the cryoTEM sample preparation, which is currently one of our interest areas for further research. Nanoparticles, dendrimers, and micelles all find wide applications in fields such as optically or magnetically active materials, and the superstructures presented here might provide $2 \mathrm{D}$ superlattices with emerging properties related to the ordering of the nanoscale building blocks, for which in nature occurring patterns can serve as inspiration. The presented strategy offers a plethora of variations in supramolecular building blocks to investigate hierarchical organization, pattern formation, size sorting, and ordering mechanisms at the nanoscale, and we anticipate our work will inspire exploration of alternative avenues for fabrication of metamaterials, e.g., thin film nonperiodic superlattices with concomitant emerging properties. ${ }^{31,35-41}$ The parallels with the study of ordered structures found in nature, ${ }^{1,2,19,20}$ in botany described as phyllotaxis, inspired us to define the current field of study as nanotaxis.

\section{EXPERIMENTAL SECTION}

Materials. Amine-terminated polyamidoamino dendrimers generation 7-9 were obtained from Dendritech Inc., MI, USA, as 5 wt \% methanolic solutions. $\mathrm{pMAA}_{64}-b-\mathrm{PEO}_{885}\left(M_{\mathrm{w}} / M_{\mathrm{n}}=1.15\right)$ was obtained from Polymer Sources Inc., Canada, and used as a $5 \mathrm{mM}$ solution based on carboxylic acid content. $\mathrm{HAuCl}_{4} \cdot 3 \mathrm{H}_{2} \mathrm{O}$ was obtained from TCI. (3-(N-Morpholino)propanesulfonic acid) (MOPS), $\mathrm{NaBH}_{4}, 1 \mathrm{M} \mathrm{HCl}$, and $1 \mathrm{M} \mathrm{NaOH}$ solutions were obtained from Sigma-Aldrich. NaOD, DCl, and $\mathrm{D}_{2} \mathrm{O}$ with a purity of $99.96 \% \mathrm{D}$ were obtained from Eurisotop, France.

Apparatus. DLS was done on a Malvern Zetasizer Nano S equipped with a laser operating at $633 \mathrm{~nm}$. Sample grids for electron microscopy were obtained from Electron Microscopy Sciences (EMS, Hatfield, PA, USA) and were rendered hydrophilic using a plasma cleaning setup ( $15 \mathrm{~s}$ at $10^{-1}$ Torr). For cryoTEM, samples were cast on Quantifoil R2/2 grids or 400 mesh holey carbon grids. After 
blotting, samples were plunged into liquid ethane using a Vitrobot system (FEI Company). Samples were imaged at $\sim 90 \mathrm{~K}$ in a JEOL 2100 TEM operating at $200 \mathrm{kV}$. For normal TEM, solutions were deposited on hydrophilic 400 mesh carbon-coated copper grids. The micrographs shown are representative for grid holes at different locations on the TEM grids and for samples made on different days. TEM image analyses were done using custom MATLAB scripts (The MathWorks Inc., Natick, MA, USA) and FIJI (https://fiji.sc/).

Synthesis of Dendrimer-Encapsulated Nanoparticles. G7$\mathrm{Au}_{256} \mathrm{DENs}$ were made following established protocols. ${ }^{15}$ Briefly, 50 $\mu \mathrm{L}(17 \mathrm{nmol})$ of $5 \mathrm{wt} \%$ PAMAM G7- $\mathrm{NH}_{2}$ in methanol was transferred to a $5 \mathrm{~mL}$ vial, and the solvent was evaporated under reduced pressure. Next, $2 \mathrm{~mL}$ of water was added to dissolve the PAMAM, and the $\mathrm{pH}$ was adjusted to 3 using $1 \mathrm{M} \mathrm{HCl}$, after which 256 molar equiv of $\mathrm{Au}^{3+}$ to PAMAM were added as $1 \mathrm{~mL}$ of a $4.4 \mathrm{mM}$ aqueous solution of $\mathrm{HAuCl}_{4}$ at $\mathrm{pH} 3$. The resulting solution was then stirred for 20 min, after which $44 \mu \mathrm{L}$ of a $1 \mathrm{M}$ solution of $\mathrm{NaBH}_{4}$ in 0.3 $\mathrm{M} \mathrm{NaOH}\left(10\right.$ molar equiv to $\left.\mathrm{Au}^{3+}\right)$ was added. This resulted in the reduction of $\mathrm{Au}^{3+}$ to AuDENs, indicated by the change from colorless to a dark brown solution within seconds after addition. After reduction, the $\mathrm{pH}$ was set to 7 using $\mathrm{HCl}$, and the $\mathrm{Au}_{256}$ DENS were stored at $4{ }^{\circ} \mathrm{C}$ as a $5.6 \mu \mathrm{M}$ solution. The G8 and G9 DENs were made in a similar way, using 512 and 1024 equivalents, respectively, of $\mathrm{Au}:$ PAMAM- $\mathrm{NH}_{2}$.

Dendrimer-Encapsulated Nanoparticles in Micelles. To obtain dendrimicelles under charge stoichiometric conditions, $20 \mu \mathrm{L}$ of GX DENs ( $\sim 58 \mathrm{nmol}$ positive charge based on surface groups, GX being generation 7, 8, or 9 PAMAM) was dissolved in $149 \mu \mathrm{L}$ of water, and $20 \mu \mathrm{L}$ of $0.2 \mathrm{M}$ MOPS buffer at $\mathrm{pH} 7.0$ was added. Then, $11 \mu \mathrm{L}$ of pMAA $_{64}-b-\mathrm{PEO}_{885}(55 \mathrm{nmol}$ based on $-\mathrm{COOH})$ was added, and the sample was sonicated for $2 \mathrm{~min}$. To obtain more polydisperse micelles off-stoichiometric charge fractions were used. Here, the ratio of block copolymer to dendrimer was adjusted accordingly, keeping the final volume constant at $200 \mu \mathrm{L}$. Samples were left to equilibrate for at least 1 day before characterization using cryoTEM.

Image Segmentation. To allow for an in-depth analysis of the individual micelle characteristics within the images, a semiautomatic micelle segmentation protocol was developed. This segmentation consisted of three main sections. First, an automatic intensity-based thresholding was applied to detect the gold nanoparticles, located within the micelles. Second, the detected features were filtered by size, excluding small noise structures, dilated, and connected based on interparticle distance. This generated filled circular structures, approximating the actual micelles in the original image. Lastly, manual adjustment and verification of the created micelle segmentation with respect to the original microscopy image provided the final segmentation output. After the segmentation, every segmented blob was digitally labeled and characterized for its individual properties, e.g., micelle centroid location, micelle area in the image, and micelle diameter. These values were used to create heat map images, visualizing the micelle distributions based on their area. For these images, perfectly round circles, with the appropriate diameter, were drawn at the micelle centroid locations, using a linear color coding to depict the size distribution. Micelle coordinates were converted to polar coordinates using the approximated center of the pattern. The nearest neighbors of a dendrimicelle were defined as the particles that have their centroid coordinates located within a circle with a radius of 1.5 times the distance of a dendrimicelle and its closest neighbor.

Dendrimicelle Core Packing Density. The dendrimicelle core packing density was calculated by dividing the core volume by the total dendrimer volume inside the core. The total dendrimer volume inside the dendrimicelle core was calculated by multiplying the number of dendrimers per dendrimicelle by the dendrimer volume. The dendrimer volume was calculated from the manufacturer's specified diameter (8.1, 9.7, and $11.4 \mathrm{~nm}$ for G7, G8, and G9 PAMAM dendrimers, respectively), assuming the dendrimers to be spherical.

\section{ASSOCIATED CONTENT}

\section{Supporting Information}

The Supporting Information is available free of charge on the ACS Publications website at DOI: 10.1021/acsnano.7b05541.

DLS and TEM characterization of PAMAM G7- $\mathrm{Au}_{256}$, $\mathrm{G} 8-\mathrm{Au}_{512}$, and $\mathrm{G} 9-\mathrm{Au}_{1024}$ as well as enlarged cryoTEM micrographs and analyses (PDF)

\section{AUTHOR INFORMATION}

\section{Corresponding Author}

*E-mail: aldrik.velders@wur.nl.

ORCID

Jan Bart ten Hove: 0000-0002-0097-2147

Aldrik H. Velders: 0000-0002-6925-854X

Notes

The authors declare no competing financial interest.

\section{ACKNOWLEDGMENTS}

Jan van Lent and Marcel Giesbers of the Wageningen Electron Microscopy Centre are thanked for their help with (cryo) electron microscopy. J.B.t.H. and A.H.V. thank NWO for financial support of the MONALISA project 717.013.006. The research leading to these results has received funding from the European Research Council (ERC) under the European Union's Seventh Framework Program FP7/2007-2013 (Grant No. 2012-306890) and the Horizon 2020 Research and Innovation Programme under the Marie Sklodowska-Curie grant agreement no. 642192 .

\section{REFERENCES}

(1) Thompson, D. W. On Growth and Form; University Press: Cambridge, 1917

(2) Ball, P. Shapes: Nature's Patterns; Oxford University Press Inc., New York, 2009.

(3) Whitesides, G. M.; Grzybowski, B. Self-Assembly at All Scales. Science 2002, 295, 2418-2421.

(4) Boles, M. A.; Engel, M.; Talapin, D. V. Self-Assembly of Colloidal Nanocrystals: From Intricate Structures to Functional Materials. Chem. Rev. 2016, 116, 11220-11289.

(5) Li, M.; Schnablegger, H.; Mann, S. Coupled Synthesis and SelfAssembly of Nanoparticles to Give Structures with Controlled Organization. Nature 1999, 402, 393-395.

(6) Zeng, C. J.; Chen, Y. X.; Kirschbaum, K.; Lambright, K. J.; Jin, R. C. Emergence of Hierarchical Structural Complexities in Nanoparticles and Their Assembly. Science 2016, 354, 1580-1584.

(7) Zeng, X. B.; Ungar, G.; Liu, Y. S.; Percec, V.; Dulcey, S. E.; Hobbs, J. K. Supramolecular Dendritic Liquid Quasicrystals. Nature 2004, 428, 157-160.

(8) Wang, Y. Z.; Zeiri, O.; Raula, M.; Le Ouay, B.; Stellacci, F.; Weinstock, I. A. Host-Guest Chemistry with Water-Soluble Gold Nanoparticle Supraspheres. Nat. Nanotechnol. 2017, 12, 170-176.

(9) Zeng, X. B.; Kieffer, R.; Glettner, B.; Nurnberger, C.; Liu, F.; Pelz, K.; Prehm, M.; Baumeister, U.; Hahn, H.; Lang, H.; Gehring, G. A.; Weber, C. H.; Hobbs, J. K.; Tschierske, C.; Ungar, G. Complex Multicolor Tilings and Critical Phenomena in Tetraphilic Liquid Crystals. Science 2011, 331, 1302-1306.

(10) Hernandez-Garcia, A.; Kraft, D. J.; Janssen, A. F. J.; Bomans, P. H. H.; Sommerdijk, N.; Thies-Weesie, D. M. E.; Favretto, M. E.; Brock, R.; de Wolf, F. A.; Werten, M. W. T.; van der Schoot, P.; Stuart, M. A. C.; de Vries, R. Design and Self-Assembly of Simple Coat Proteins for Artificial Viruses. Nat. Nanotechnol. 2014, 9, 698-702.

(11) Voets, I. K.; de Keizer, A.; Stuart, M. A. C. Complex Coacervate Core Micelles. Adv. Colloid Interface Sci. 2009, 147-48, 300-318. 
(12) Astruc, D.; Boisselier, E.; Ornelas, C. Dendrimers Designed for Functions: From Physical, Photophysical, and Supramolecular Properties to Applications in Sensing, Catalysis, Molecular Electronics, Photonics, and Nanomedicine. Chem. Rev. 2010, 110, 1857-1959.

(13) Tomalia, D. A.; Khanna, S. N. A Systematic Framework and Nanoperiodic Concept for Unifying Nanoscience: Hard/Soft Nanoelements, Superatoms, Meta-Atoms, New Emerging Properties, Periodic Property Patterns, and Predictive Mendeleev-Like Nanoperiodic Tables. Chem. Rev. 2016, 116, 2705-2774.

(14) Wang, J. Y.; Velders, A. H.; Gianolio, E.; Aime, S.; Vergeldt, F. J.; Van As, H.; Yan, Y.; Drechsler, M.; de Keizer, A.; Stuart, M. A. C.; van der Gucht, J. Controlled Mixing of Lanthanide(III) Ions in Coacervate Core Micelles. Chem. Commun. 2013, 49, 3736-3738.

(15) Ten Hove, J. B.; Wang, J.; Van Leeuwen, F. W. B.; Velders, A. H. Dendrimer-Encapsulated Nanoparticle-Core Micelles as Versatile Strategy for Particle-in-a-Box-in-a-Box Nanostructures. Nanoscale 2017, DOI: $10.1039 / \mathrm{c} 7 \mathrm{nr} 06773$.

(16) Crooks, R. M.; Zhao, M. Q.; Sun, L.; Chechik, V.; Yeung, L. K. Dendrimer-Encapsulated Metal Nanoparticles: Synthesis, Characterization, and Applications to Catalysis. Acc. Chem. Res. 2001, 34, 181190.

(17) Gomez, M. V.; Guerra, J.; Myers, V. S.; Crooks, R. M.; Velders, A. H. Nanoparticle Size Determination by ${ }^{1} \mathrm{H}$ NMR Spectroscopy. J. Am. Chem. Soc. 2009, 131, 14634-14635.

(18) Gomez, M. V.; Guerra, J.; Velders, A. H.; Crooks, R. M. Nmr Characterization of Fourth-Generation Pamam Dendrimers in the Presence and Absence of Palladium Dendrimer-Encapsulated Nanoparticles. J. Am. Chem. Soc. 2009, 131, 341-350.

(19) Mitchison, G. J. Phyllotaxis and Fibonacci Series. Science 1977, 196, 270-275.

(20) Vogel, H. Better Way to Construct the Sunflower Head. Math. Biosci. 1979, 44, 179-182.

(21) Grohn, F.; Bauer, B. J.; Akpalu, Y. A.; Jackson, C. L.; Amis, E. J. Dendrimer Templates for the Formation of Gold Nanoclusters. Macromolecules 2000, 33, 6042-6050.

(22) Ungar, G.; Zeng, X. B. Frank-Kasper, Quasicrystalline and Related Phases in Liquid Crystals. Soft Matter 2005, 1, 95-106.

(23) Danino, D. Cryo-Tem of Soft Molecular Assemblies. Curr. Opin. Colloid Interface Sci. 2012, 17, 316-329.

(24) Friedrich, H.; Frederik, P. M.; de With, G.; Sommerdijk, N. Imaging of Self-Assembled Structures: Interpretation of Tem and Cryo-Tem Images. Angew. Chem., Int. Ed. 2010, 49, 7850-7858.

(25) Glaeser, R. M.; Han, B. G.; Csencsits, R; Killilea, A.; Pulk, A.; Cate, J. H. D. Factors That Influence the Formation and Stability of Thin, Cryo-Em Specimens. Biophys. J. 2016, 110, 749-755.

(26) Ershov, D.; Sprakel, J.; Appel, J.; Stuart, M. A. C.; van der Gucht, J. Capillarity-Induced Ordering of Spherical Colloids on an Interface with Anisotropic Curvature. Proc. Natl. Acad. Sci. U. S. A. 2013, 110, 9220-9224.

(27) Iacovella, C. R; Keys, A. S.; Glotzer, S. C. Self-Assembly of SoftMatter Quasicrystals and Their Approximants. Proc. Natl. Acad. Sci. U. S. A. 2011, 108, 20935-20940.

(28) Li, C.; Zhang, X.; Cao, Z. Triangular and Fibonacci Number Patterns. Science 2005, 309, 909-911.

(29) Lin, Y.; Boker, A.; Skaff, H.; Cookson, D.; Dinsmore, A. D.; Emrick, T.; Russell, T. P. Nanoparticle Assembly at Fluid Interfaces: Structure and Dynamics. Langmuir 2005, 21, 191-194.

(30) Mueggenburg, K. E.; Lin, X. M.; Goldsmith, R. H.; Jaeger, H. M. Elastic Membranes of Close-Packed Nanoparticle Arrays. Nat. Mater. 2007, 6, 656-660.

(31) Vanmaekelbergh, D. Self-Assembly of Colloidal Nanocrystals as Route to Novel Classes of Nanostructured Materials. Nano Today 2011, 6, 419-437.

(32) Dotera, T.; Oshiro, T.; Ziherl, P. Mosaic Two-Lengthscale Quasicrystals. Nature 2014, 506, 208-211.

(33) Ziherl, P.; Kamien, R. D. Maximizing Entropy by Minimizing Area: Towards a New Principle of Self-Organization. J. Phys. Chem. B 2001, 105, 10147-10158.
(34) Glaser, M. A.; Grason, G. M.; Kamien, R. D.; Košmrlj, A.; Santangelo, C. D.; Ziherl, P. Soft Spheres Make More Mesophases. Europhys. Lett. 2007, 78, 46004.

(35) Bigioni, T. P.; Lin, X. M.; Nguyen, T. T.; Corwin, E. I.; Witten, T. A.; Jaeger, H. M. Kinetically Driven Self Assembly of Highly Ordered Nanoparticle Monolayers. Nat. Mater. 2006, 5, 265-270.

(36) Boneschanscher, M. P.; Evers, W. H.; Geuchies, J. J.; Altantzis, T.; Goris, B.; Rabouw, F. T.; van Rossum, S. A. P.; van der Zant, H. S. J.; Siebbeles, L. D. A.; Van Tendeloo, G.; Swart, I.; Hilhorst, J.; Petukhov, A. V.; Bals, S.; Vanmaekelbergh, D. Long-Range Orientation and Atomic Attachment of Nanocrystals in 2D Honeycomb Superlattices. Science 2014, 344, 1377-1380.

(37) Cargnello, M.; Johnston-Peck, A. C.; Diroll, B. T.; Wong, E.; Datta, B.; Damodhar, D.; Doan-Nguyen, V. V. T.; Herzing, A. A.; Kagan, C. R.; Murray, C. B. Substitutional Doping in Nanocrystal Superlattices. Nature 2015, 524, 450-453.

(38) Dong, A. G.; Chen, J.; Vora, P. M.; Kikkawa, J. M.; Murray, C. B. Binary Nanocrystal Superlattice Membranes Self-Assembled at the Liquid-Air Interface. Nature 2010, 466, 474-477.

(39) Huang, X. Q.; Tang, S. H.; Mu, X. L.; Dai, Y.; Chen, G. X.; Zhou, Z. Y.; Ruan, F. X.; Yang, Z. L.; Zheng, N. F. Freestanding Palladium Nanosheets with Plasmonic and Catalytic Properties. Nat. Nanotechnol. 2011, 6, 28-32.

(40) Parker, G. J. Biomimetically-Inspired Photonic Nanomaterials. J. Mater. Sci.: Mater. Electron. 2010, 21, 965-979.

(41) Ungar, G.; Liu, Y. S.; Zeng, X. B.; Percec, V.; Cho, W. D. Giant Supramolecular Liquid Crystal Lattice. Science 2003, 299, 1208-1211. 